\title{
INTRODUCTION
}

\section{Winners of the ERS Annual Awards 2006}

\author{
P.S. Burge and V.C. Moore
}

$\mathbf{T}$ he European Respiratory Review (ERR) is delighted to publish articles from winners of European Respiratory Society (ERS) Annual Awards 2006. The awardees were invited to include the background of their work, how they thought it might be important to respiratory medicine, the aims of the team in which they work and to include their award-winning abstract. The articles encompass a spectrum of research and have been split into three sections, presenting scientific articles in the first and third sections, and clinical articles in the second section. They provide an insight into research being carried out around the world today.

\section{SECTION 1}

The first chapter presents Dr Stephen A. Renshaw's research: "The molecular controls of resolution of inflammation: what can we learn from zebrafish?" (page 168). His PhD thesis explored a family of death receptors on neutrophil surfaces and their signalling pathways. This became difficult in human neutrophils, so the zebrafish has now become the in vivo model for resolving inflammation, as it shares many features of the human immune system and has helped the team to generate fluorescent systems for the easy visualisation of neutrophilic inflammation in vivo.

Dr Emma H. Baker's primary research focus is on respiratory epithelial transport processes, but through collaboration with Dr Peterson, she undertook the study entitled "Development of a biomarker for lung inflammation in COPD [chronic obstructive pulmonary disease] through analysis of labelled leukocyte transit through the lung circulation" (page 170), investigating the ratio of the downslope of curve in the lungs compared with the heart. She found a significantly smaller ratio in stable COPD patients compared to controls.

Dr Hajime Yoshisue worked at the University of Southampton's School of Medicine, Southampton, UK, on his project entitled "Molecular mechanisms of the synergy between cysteinyl-leukotrienes and receptor tyrosine kinase growth factors on human bronchial fibroblast proliferation" (page 172), finding that the effect of cysteinyl-leukotrienes involves a pertussis toxin-sensitive and protein kinase C-mediated intracellular pathway, leading to sustained growth factor-dependent phosphorylation of extracellular signal-regulated kinase $1 / 2$ and Akt.

The research in the department in which Charles Pilette works focuses on the role of $\operatorname{IgA}$ and mucosal immunity in airway responses. His winning abstract (page 175) found that secretory component production increased when there was an intermediate neutrophil number versus control, but decreased when there were high neutrophil numbers, suggesting a dual effect of neutrophils on epithelial secretory component.

Dr Stefan Pabst works as a ward and outpatient physician at the University Hospital of Bonn, Bonn, Germany, and is interested in the aetiology of COPD, sarcoidosis and obstructive sleep apnoea. His abstract entitled "A stop codon polymorphism of toll-like receptor 5 is associated with a stable course of chronic obstructive lung disease" (page 177) suggests that theTLR $5^{392 S T O I}$ polymorphism is associated with a stable course of COPD.

Dr Melanie Königshoff is currently undertaking a $\mathrm{PhD}$ at the University of Giessen School of Medicine (Giessen, Germany) focusing on the function and interaction of alveolar epithelial cells and interstitial fibroblasts. Her abstract entitled "Wnt-inducible protein (WISP-1) is a key regulator of alveolar epithelial cell hyperplasia in pulmonary fibrosis" (page 180) revealed gene regulatory networks in lung fibrosis by comparing gene expression profiles of alveolar epithelial cells in mice.

Dr Suzanne Zuyderduyn from Leiden University Medical Center, Leiden, the Netherlands, presented the "Role of purinergic receptors in the activation of human airway smooth muscle cells by the antimicrobial peptide LL-37"' (page 182). The main research spotlight of the department in which she works concerns airway smooth muscle and its role in the remodelling of airways and airway inflammation. Her research showed that the human cathelicidin peptide LL-37 enhanced interleukin-8 release from airway smooth muscle and this is mediated by purinergic receptors,
AFFILIATIONS

Dept of Respiratory Medicine, Birmingham Heartlands Hospital, Birmingham, UK

CORRESPONDENCE

V.C. Moore

Dept of Respiratory Medicine Birmingham Heartlands Hospital Bordesley Green East Birmingham B9 5SS UK Fax: 441217720292 E-mail: vicky.c.moore@ heartofengland.nhs.uk 
extracellular signal-regulated kinase $1 / 2$ activation and p38 and Src signalling.

\section{SECTION 2}

The next group of articles is led by Penny Marno from the Health Forecasting team at the UK's meteorological service. Her abstract entitled "How different measures of cold weather affect chronic obstructive pulmonary disease (COPD) hospital admissions in London" (page 185) describes how this phenomena was investigated due to the strong correlations found between the weather and COPD, and the development of their COPD forecasting model. The research concluded that wind chill had the strongest correlation, with a 1-week lag accounting for $20 \%$ of the variation in admissions. Overall, all measures of cold showed significant correlations.

Dr Sarah J. Deacon from the Institute for Lung Health, Glenfield Hospital, Leicester, UK, investigated the improvement of physical performance in COPD, as it is an important therapeutic goal. As there has been evidence that phosphocreatinine levels are lower in COPD patients than in healthy subjects, this may represent a potential therapeutic target. Her abstract, entitled "Does creatine supplementation enhance the effects of physical training during pulmonary rehabilitation in COPD?" (page 187), did not find any enhanced benefits over pulmonary rehabilitation alone.

Dr Sundeep Kaul works as a Clinical Research Fellow in the Muscle Laboratory, Respiratory Medicine, Kings College Hospital, London, UK. His study was undertaken in stable COPD patients and is entitled "The effect of entrainment site and inspiratory pressure on the delivery of oxygen therapy during non-invasive mechanical ventilation (NIMV) in acute COPD patients" (page 190). He found that the addition of oxygen to the mask at low inspiratory pressures resulted in higher oxygen delivery.

Jocimar A. Martins works at Arnaldo Gavazza Filho Hospital, Ponte Nova, Brazil, as the Head of Service in Physiotherapy. Her abstract entitled "The effects of the ELTGOL on mucociliary clearance in patients with COPD" (page 192) was performed on 12 patients with COPD and found that expiration with the glottis open in lateral posture (ELTGOL) significantly increased mucociliary clearance.

Dr Stephan Budweiser from Donaustauf Hospital, Donaustauf, Germany, is involved in a number of scientific projects investigating chronic respiratory failure patients. His abstract entitled "Base excess, a marker of chronic hypercapnic respiratory failure and predictor of survival in COPD" (page 194) describes the discovery that base excess is an independent predictor of mortality in these patients and that base excess changes when chronic hypercapnic respiratory failure is reversed.

In 2003 at Hokkaido University Hospital (Sapporo, Japan), Dr Masaru Hasegawa started working on a COPD cohort designed to investigate the natural history of COPD according to phenotype. His research, entitled "Relationship of airway dimensions with airflow limitation or lung volumes in chronic obstructive pulmonary disease (COPD)" (page 197) concluded that the forced expiratory volume in one second (FEV1) per cent predicted and residual volume/total lung capacity correlated with airway luminal area and, to some extent, with wall thickening between the third and sixth generations.

Dr Russell P. Bowler is the Director of the COPD Clinic at the National Jewish Medical and Research Centre (Denver, CO, USA). The laboratory based at the centre is involved with discovering biomarkers for COPD using genetic and proteomic approaches. His abstract entitled "Extracellular superoxide dismutase (EC-SOD) as a protective factor for risk of chronic obstructive pulmonary disease" (page 200) showed that the presence of elevated levels of extracellular superoxide dismutase partially predicted a resistance to the effects of smoking (decreased forced expiratory volume in one second (FEV1)) in smokers.

Dr Silvia Carraro works at the Dept of Paediatrics in the Respiratory Medicine and Allergy Unit at the University of Padova, Padova, Italy. Dr Carraro won an ERS Annual Award for Paediatric Respiratory Research in Europe, and is one of two authors in this issue of the ERR awarded for an oral presentation given at the 2006 ERS Congress. Her work, entitled "Noninvasive markers of airway inflammation and redox biochemistry applied to asthma" (page 202), is mainly focused on exhaled nitric oxide levels and oxidative stress markers in exhaled breath condensate; raised levels of 8isoprostane, 3-nitrotyrosine and cysteinyl-leukotrienes have been found in asthmatic children.

Lastly, Cleo C. van Diemen from the Dept of Epidemiology, University of Groningen, Groningen, the Netherlands, was awarded two prizes. Her first was from the Occupational and Epidemiology Assembly for an abstract entitled "A Disintegrin and Metalloprotease 33 polymorphisms and lung function decline in the general population" (page 204), which concluded that $\mathrm{A}$ disintegrin and metalloprotease 33 (ADAM33) and single nucleotide polymorphisms are associated with accelerated lung function decline in the general population. Her second award-winning abstract entitled "Polymorphisms in surfactant proteins are associated with FEV1 decline and development of COPD in the general population" (page 204) was along a similar theme, finding that surfactant protein-D Met11Thr single nucleotide polymorphism is associated with a faster decline in FEV1 and COPD development in the general population.

\section{SECTION 3}

The last group of articles begins with Dr Juanita H. J. Vernooy, a postdoctoral fellow at Maastricht University, Maastricht, the Netherlands, whose current research project started in March 2006 and centred on leptin in innate immunity in the lung. A mixture of in vitro and in vivo disease models was used, and Dr Vernooy's abstract entitled "Suppressed pulmonary expression of leptin in lipopolysaccharide-induced acute and chronic lung inflammation" (page 207) focused on male Swiss mice. She found that chronic lung inflammation resulted in complete suppression of pulmonary leptin expression.

Marta Díez presented "Plasticity of vascular progenitor cells: implications in pulmonary vascular remodelling in COPD" (page 209). Her work in the Pneumology Service, Hospital Clinic, Barcelona, Spain, assesses whether the number of circulating progenitors in COPD patients correlates with vascular function impairment. She found that vascular 
progenitor cells have the potential to differentiate in vitro into smooth muscle cells and that this could contribute to pulmonary vascular remodelling.

Dr Ellen M. Drost's research centres on the mechanisms of lung disease caused by inhaled air pollutants. Her abstract "Diminished peroxisome proliferator-activated receptor (PPAR) regulation as a potential mechanism for the persistent inflammation in chronic obstructive pulmonary disease" (page 211) describes the use of in vitro assays to assess the effect of PPAR agonist treatment on inflammatory cytokine generation; a lesser inflammatory response to TNF- $\alpha$ was found.

Dr Kay Roy is pursuing a PhD in the role of endogenous nitric oxide in COPD airway inflammation in Manchester, UK. Her abstract "Multiple flow rate modelling of nitric oxide in COPD - methodological concerns" (page 213) showed that smoking reduced the airway wall concentration of nitric oxide but had no effect on airway wall diffusing capacity or alveolar concentration.

Dr Mariëlle Pijnenburg also investigated aspects of nitric oxide in her research entitled "Exhaled nitric oxide in childhood asthma: methodological issues and clinical applications" (page 215). Dr Pijnenburg won an ERS Annual Award for Paediatric Respiratory Research, and is the second of the two authors in this issue of the ERR awarded for an oral presentation given at the 2006 ERS Congress. Dr Pijnenburg undertook four studies tackling various aspects of nitric oxide analysis, as follows: 1) flow rate problems by the use of off-line collection; 2) how storage conditions of mylar balloons affect nitric oxide levels; 3) nitric oxide in healthy children; and 4) effects of spirometry and exercise on exhaled nitric oxide in asthmatic children.

Dr Maria Pallayova from Safarik University, Kosice, Slovakia has researched into "The effect of continuous positive airway pressure on glucose excursions in diabetics with sleepdisordered breathing: the results of continuous glucose monitoring" (page 218). She applied the continuous glucose monitoring system to diabetic patients undergoing polysomnography both with and without continuous positive airway pressure (CPAP), and found that nocturnal hyperglycaemia is closely related to oxygen desaturation, with CPAP countering this effect.

Dr Silke Ryan, a Specialist Registrar at St. Vincent's University Hospital, Dublin, Ireland, presented research entitled "Predictors of elevated tumour necrosis factor $\alpha$ level in obstructive sleep apnoea syndrome" (page 221). Tumour necrosis factor (TNF)- $\alpha$ was higher in obstructive sleep apnoea patients compared to controls and this was associated with the desaturation index, the Epworth sleepiness score and total cholesterol. In an intermediate group of sleepy nonsleep apnoea patients, levels of TNF- $\alpha$ were higher than controls but lower than in the sleep apnoea patients. The severity of intermittent hypoxia was the strongest predictor.

Dr Ilonka H. van Veen is a pulmonologist at Leiden University Medical Centre, Leiden, the Netherlands, and researches into difficult-to-treat asthma. Her abstract entitled "Mannose binding lectin (MBL) levels predict lung function decline in severe asthma" (page 224) investigated serum MBL levels in severe asthmatics and prospectively determined post bronchodilator change in FEV1 over 5.7 yrs. She found that patients with high MBL levels had an increased risk of lung function decline compared to those with low levels.

Dr Trude D. Skorge is studying for a $\mathrm{PhD}$ at the University of Bergen, Bergen, Norway, analysing a longitudinal study in risk factors for incidence of respiratory symptoms. Her abstract entitled "The adult incidence of asthma and respiratory symptoms by passive smoking in utero or in childhood" (page 226), investigated the incidence of asthma and five respiratory symptoms by self-reported exposure to maternal smoking. She found that all had increased odds ratios if the mother smoked pre- or post-natally.

Dr Daiana Stolz investigated the diagnostic value of biomarkers used to diagnose infection in severely immunosuppressed patients while based at the University Hospital Basel, Basel, Switzerland. In the work entitled "Diagnostic value of procalcitonin, CRP [C-reactive protein], leukocytes and BAL [bronchoalvelar lavage] neutrophils for pulmonary complications in the immunocompromised host" (page 228), she found that BAL neutrophil counts and procalcitonin were advantageous for diagnosing bacterial infection compared to CRP and leukocyte counts.

Dilyara G. Yanbaeva works in the Genomic Department at the Institute of Biochemistry and Genetics, Ufa, Russia. She is undertaking a $\mathrm{PhD}$ involved in gene-environment interaction in diseases, such as COPD and occupational asthma. Her abstract entitled "The promoter polymorphism -1562C/T in matrix metalloproteinase-9 and COPD severity" (page 230) suggests that matrix metalloproteinase- 9 could be used as a genetic marker of COPD severity particularly in those who have early onset COPD.

We look forward to publishing articles from the 2007 winners. 